\title{
Laser Induced Breakdown Spectroscopy
}

(LIBS) and Digital Image Processing For Pigment Analysis

Amber Malloy, Megan Olsson, Shljun Tang, and Rosemarle C. Chinnl

\section{Introduction}

Art historians and art conservators are experts on visual and historical sources. While pigments, techniques, mediums, and historical documents provide substantial information about an artwork and its creator, there are some questions that experts in the field cannot answer without assistance. Pigments are more complicated in composition than they appear. Common names are often applied to pigments of the same color and do not necessarily indicate that the pigments are of the same composition. Even pigments made of the same substance can vary according to different ratios of components. The eye cannot detect such differentiations. Scientific analysis is essential to make these subtle distinctions and offer supplementary information.

Laser analysis has recently become popular for the analysis of cultural heritage objects. ${ }^{2}$ Laser Induced Breakdown Spectroscopy (LIBS) is specifically used because it is accurate, rapid, minimally destructive, and useful for depth profiling. Though it is applied primarily in laser cleaning ${ }^{3}$, it has been used for the analysis of metal artifacts, ceramics, paintings, parchment, and other culturally valuable objects. ${ }^{2}$ Such analysis can also be used for indirect dating and determining authenticity. ${ }^{4-5}$ Despite the advantages and growing popularity of LIBS, there are few examples available in the literature on its analysis of paintings.,6 Digital imaging could be used as a nondestructive method of authentication.

The purpose of this research is to investigate the usefulness of LIBS and digital imaging for the analysis of oil paint pigments for indirect dating, authentication, and identification. A series of pigments in oil medium are analyzed individually, as well as in mixtures. Samples for LIBS were prepared using 4" 4 " canvas boards. Samples for digital imaging were prepared on 1" 1 1" canvas boards and 1"x1" artists paper. All paints were professionally mixed by RGH Artist Oil Paints. Lead White, Ivory Black, Naples Yellow, Vermilion, and Ultramarine Blue were chosen to represent pigments from a traditional palette. In comparison, Titanium White, Mars Black, Cadmium Yellow, Alizarin Crimson, and Cobalt Blue were chosen to represent a modern palette.

Digital image processing technology and algorithms were used to analyze pure and mixed pigments in single and multi-laye samples. From this, histograms were created. These histograms were used to determine if there are differences
among pure and mixed pigments. The histograms were also among pure and mixed pigments. The histograms
used to determine if there are unique peak locations.

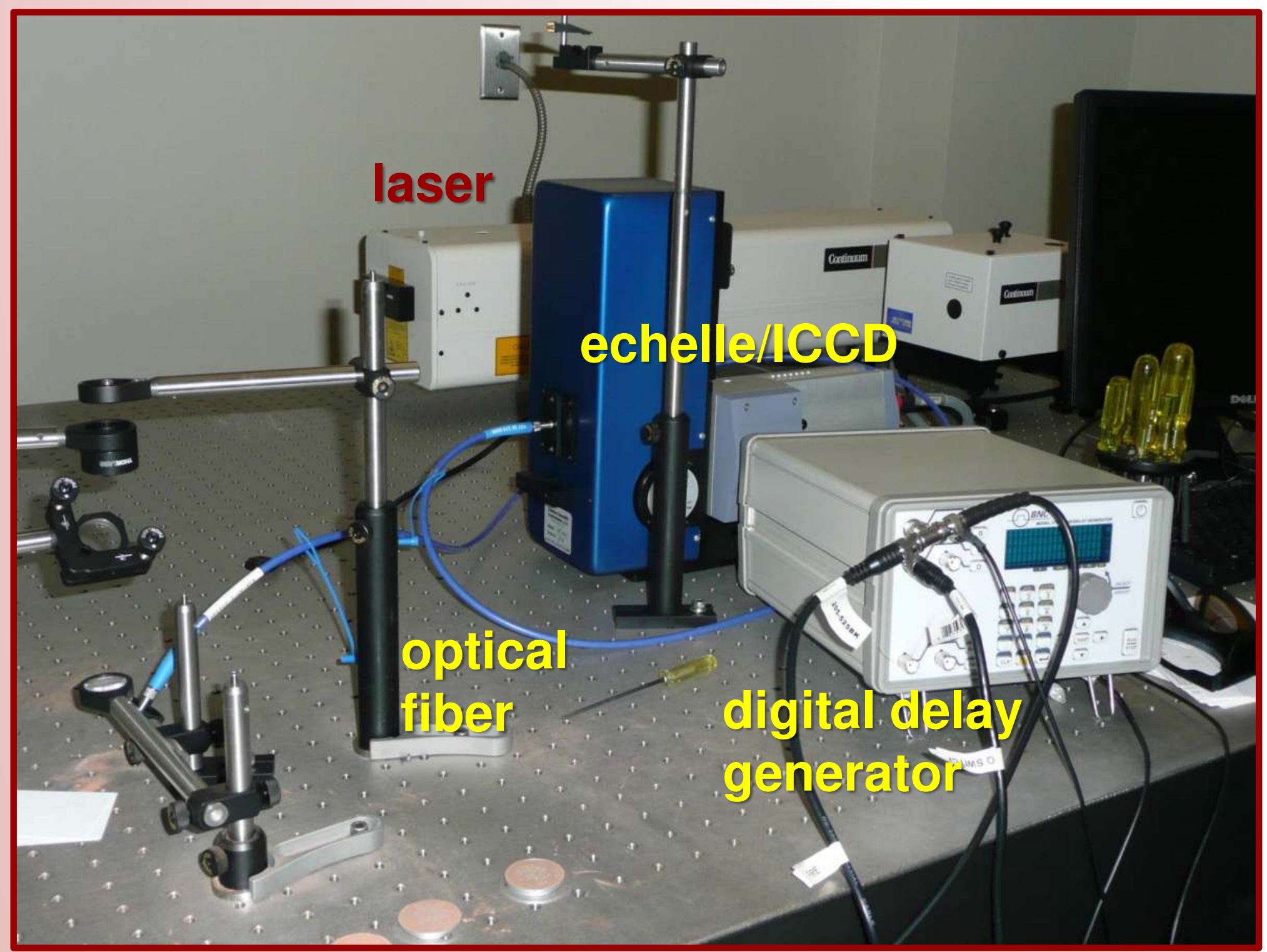

Picture of the LIBS System

In LIBS, a laser pulse is focused on a surface. This heats, ablates, atomizes, and ionizes the surface material and results in the formation of a plasma. The light emitted by the plasma is collected, spectrally resolved, and detected. Elements contained within the samples can be identified by their unique spectral signatures.

Experimental Parameters:

Laser energy: $90 \mathrm{~mJ}$

Time delay: $1 \mu \mathrm{s}$, ICCD gain: 150

0.25 second gate width: $20 \mu \mathrm{s}$; exposure; operating in either single shot mode or single shot mode with 10 accumulations

\section{Palnt Names and Type}

\section{Traditional}

Lead White

Ivory Black

Naples Yellow

Vermilion

Modern

Mars Black

Cadmium Yellow

Alizarin Crimson

Ultramarine Blue

Cobalt Blue

\section{Pletures of the Paint Samples For LIBS}
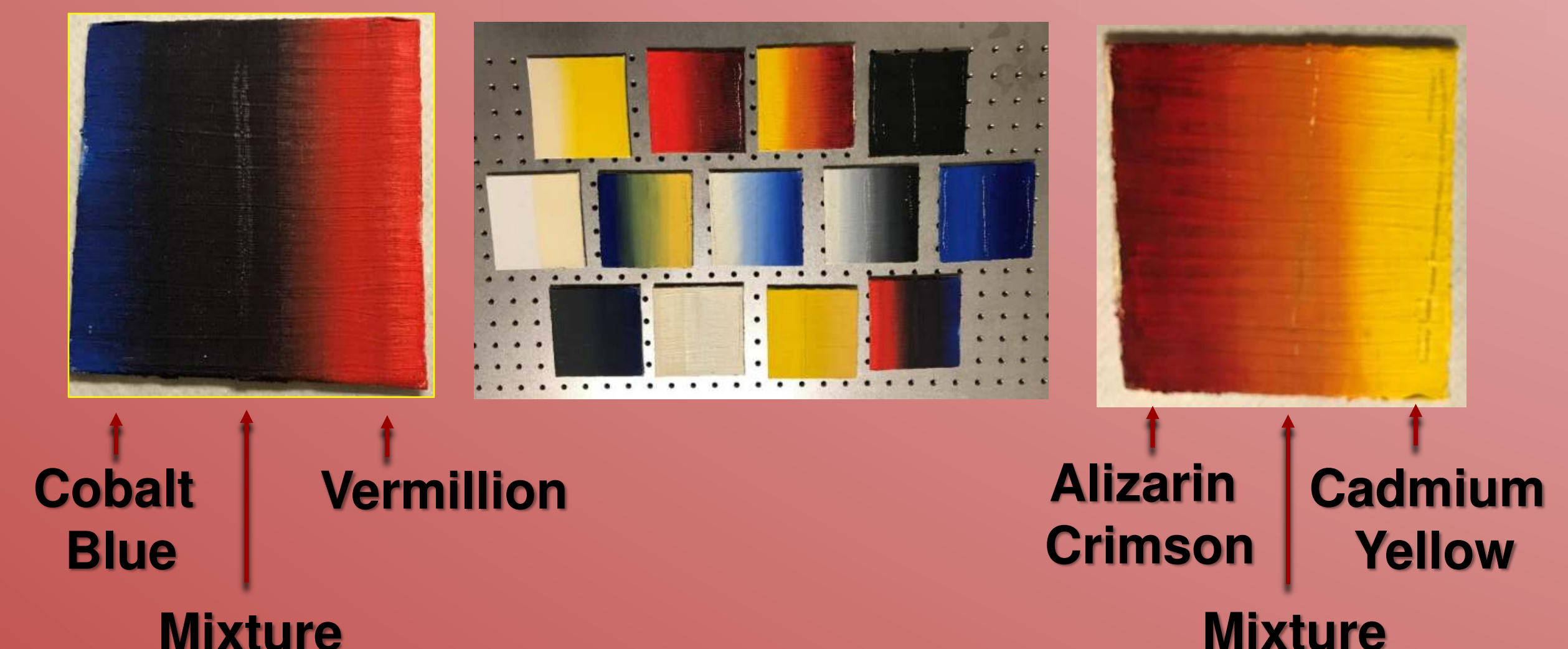

\section{Table of Emission Lines of Uniqueness}

\begin{tabular}{|c|c|c|c|}
\hline Pigment & Composition & $\begin{array}{l}\text { Identifying } \\
\text { Elements }\end{array}$ & $\begin{array}{l}\text { Wavelength } \\
\text { (nm) }\end{array}$ \\
\hline Lead White & Lead Carbonate & $\mathrm{Pb}(\mathrm{l})$ & 357.27 \\
\hline $\begin{array}{l}\text { Titanium } \\
\text { White }\end{array}$ & Titanium Dioxide & $\mathrm{Ti}(1)^{*}$ & 506.2 \\
\hline Ivory Black & Carbonized Bones & Ca (II) & 370.60 \\
\hline Mars Black & Synthetic Iron Oxide & $\mathrm{Fe}(1)^{*}$ & 407.17 \\
\hline $\begin{array}{l}\text { Naples } \\
\text { Yellow }\end{array}$ & Mixed Metal Oxide & $\mathrm{Ni}(\mathrm{l})$ & 305.08 \\
\hline $\begin{array}{l}\text { Cadmium } \\
\text { Yellow }\end{array}$ & Cadmium Sulfide & $\operatorname{Sr}(1)$ & 481.19 \\
\hline Vermillion & Mercury Sulfide & $\mathrm{Cd}(\mathrm{I})$ & 508.58 \\
\hline $\begin{array}{l}\text { Alizarin } \\
\text { Crimson }\end{array}$ & Dihydroxyanthriquinone & $\mathrm{Ca}(\mathrm{I})$ & 443.50 \\
\hline $\begin{array}{l}\text { Ultramarine } \\
\text { Blue }\end{array}$ & $\begin{array}{c}\text { Polysulphide of } \\
\text { Sodiumaluminosilicate }\end{array}$ & Si (I) & 288.16 \\
\hline Cobalt Blue & Cobalt Aluminate & Co (II) & 258.72 \\
\hline
\end{tabular}

${ }^{*}$ These atoms have many atomic lines.

\section{Pigment Identification}

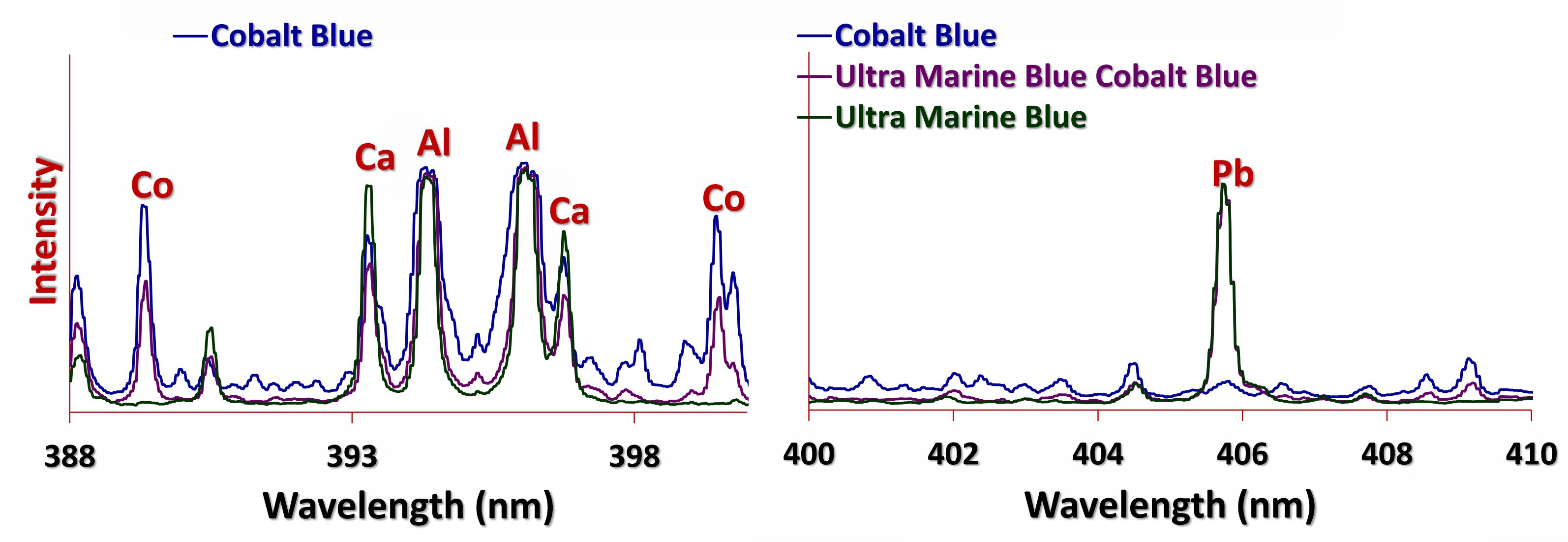

The above spectra show overlapping pure component paint spectra with a binary mixture containing both of them. Certain regions are expanded to show peaks of difference.

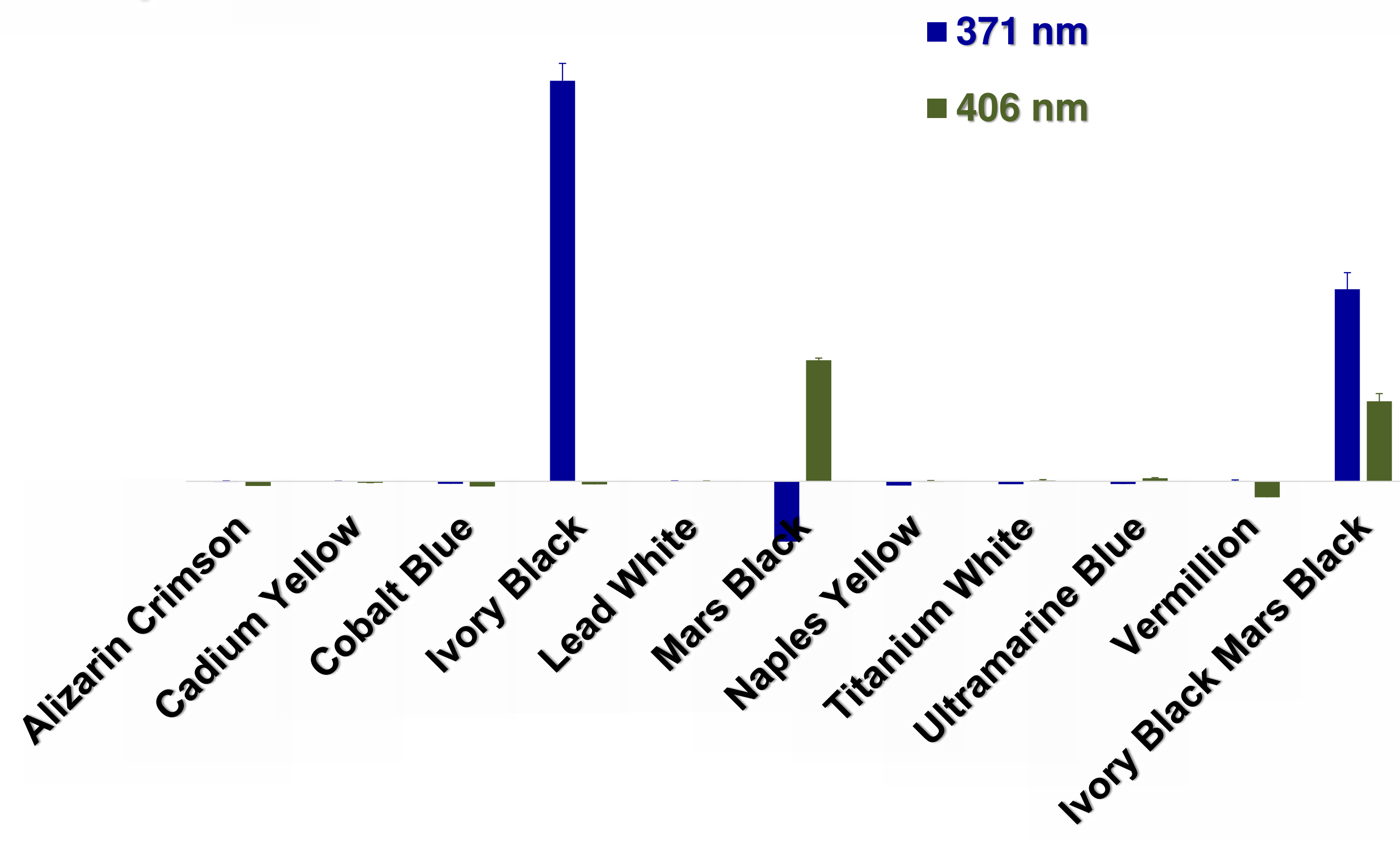

The above data shows the pure paint pigment data at two wavelengths along with one of the binary mixtures of Ivory Black and Mars Black; the two lines used for this comparison are $371.60 \mathrm{~nm}$, which is a Ca (II) line, and $407.17 \mathrm{~nm}$, which is an $\mathrm{Fe}$ (I). The data clearly shows that these two and $407.17 \mathrm{~nm}$, which is an Fe (I). The data clearly shows that these two
wavelengths can be used to predict the pure paint pigments in the mixture.

$.508 \mathrm{~nm}$

- $258 \mathrm{~nm}$ (intensityx10)

$-481 \mathrm{~nm}$

1.

- 1
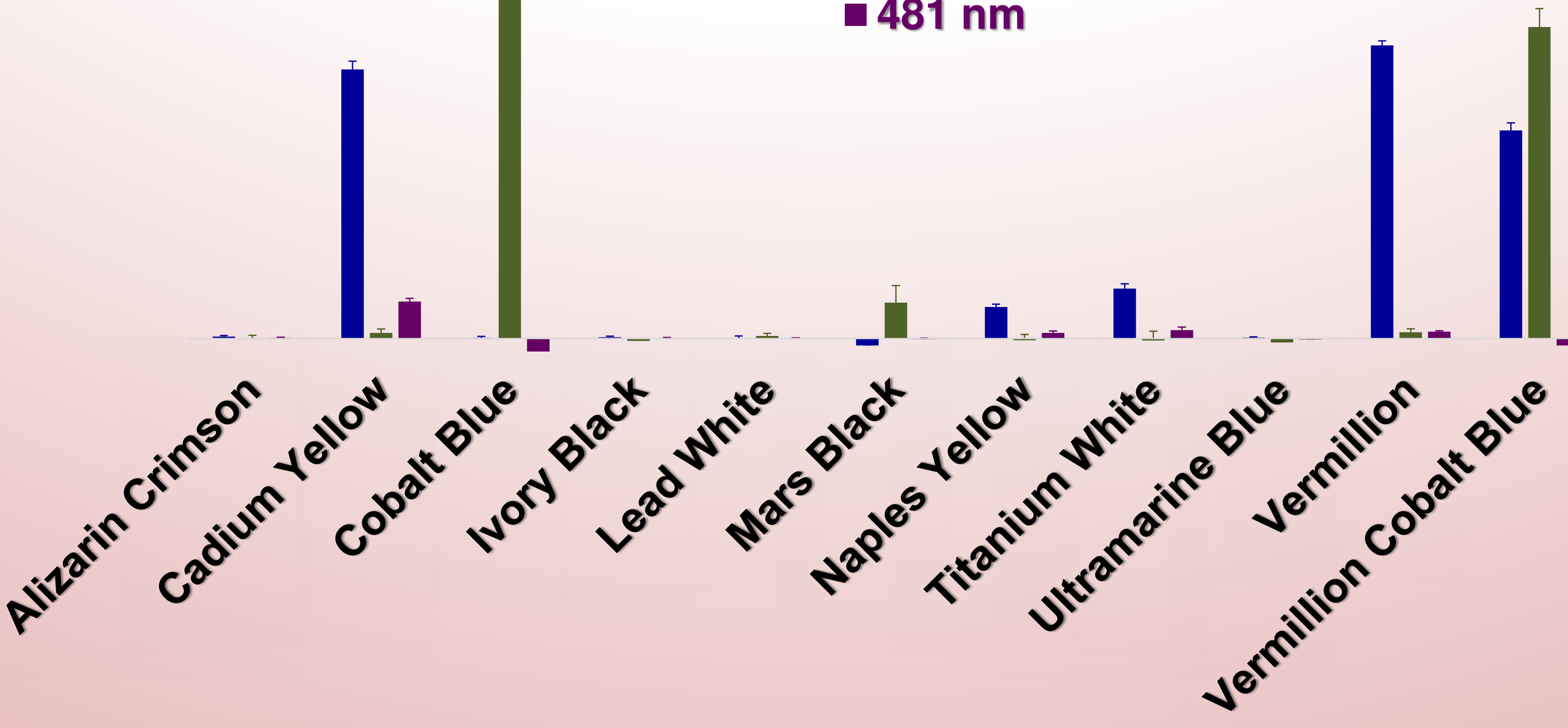

The above data shows the pure paint pigment data at two wavelengths along with one of the binary mixtures of Vermillion and Cobalt Blue; two of the lines used for this comparison are $508.58 \mathrm{~nm}$, which is a Ca (I) line, and $258.72 \mathrm{~nm}$, which is a Co (II) line, respectively. The Cobalt line was multiplied by a factor of 10 . The third line at $481.19 \mathrm{~nm}$, which is a Sr (I) line, is used to distinguish Cadmium Yellow from Vermillion is a $\mathrm{Sr}(\mathrm{I})$ line, is used to distinguish Cadmium Yellow from Vermillion
in the mixture. The data clearly shows that these three wavelengths in the mixture. The data clearly shows that these three wave
can be used to predict the pure paint pigments in the mixture.

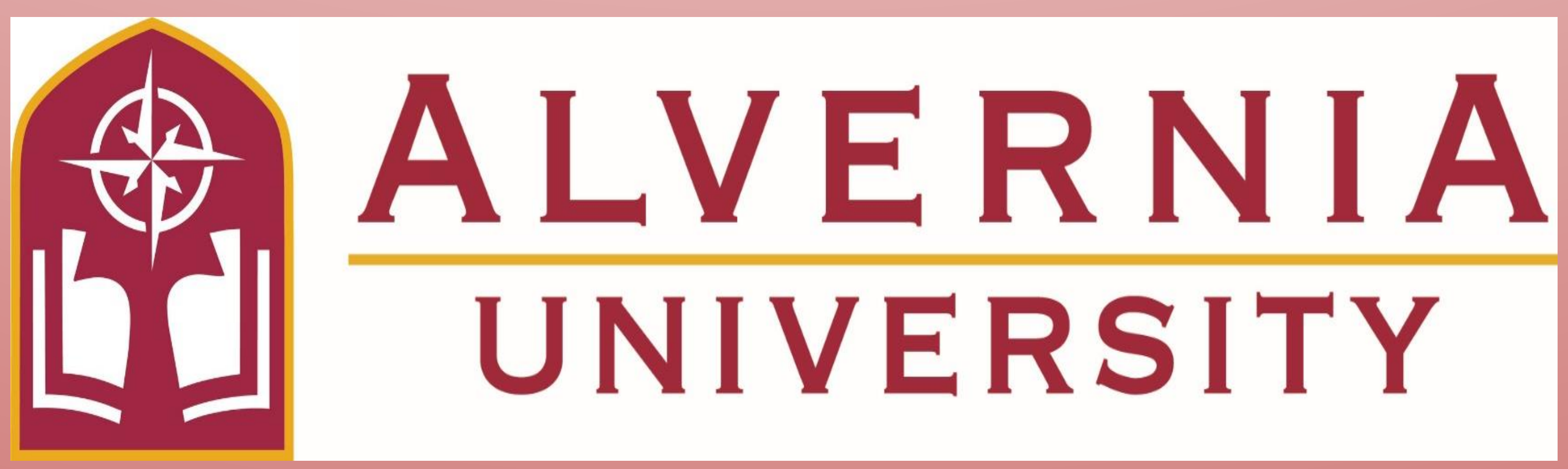

\section{Digital Imaging}

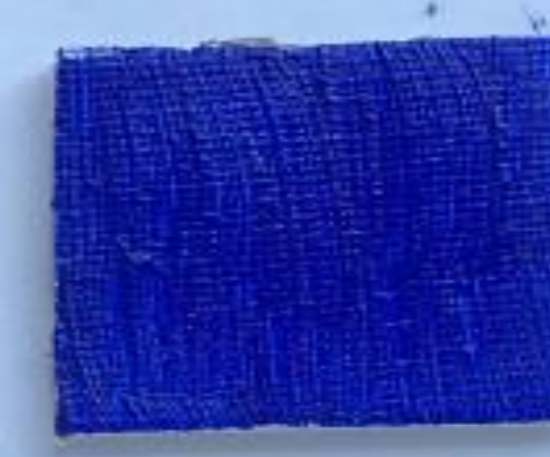

The above image shows a representative sample of the mixture Cobalt Blue and Ultramarine Blue on a 1"x1" canvas square. The same method was used for the artist paper medium. This was done for all of the pure and mixture samples.
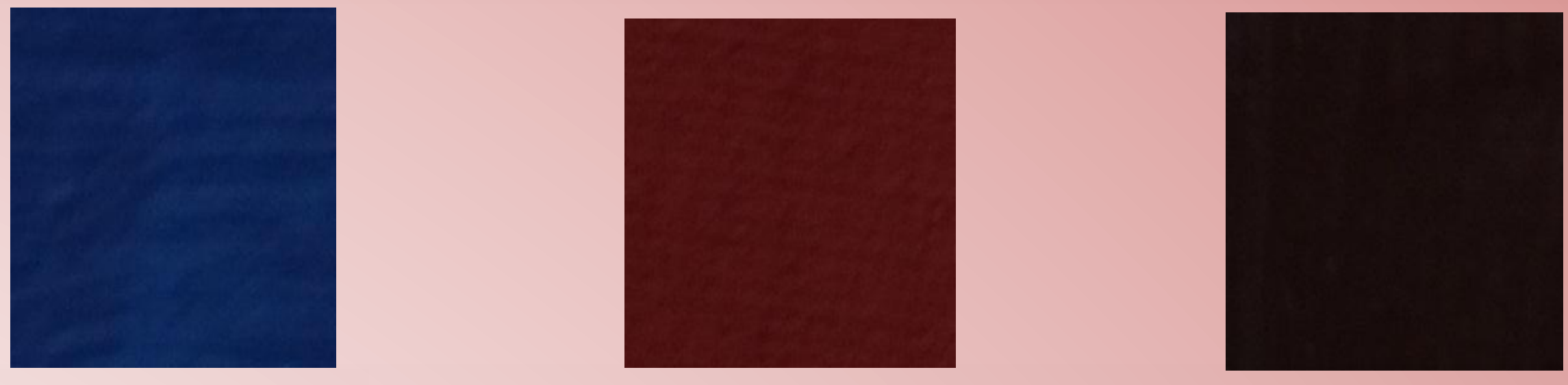

Vermillion Vermillion with Cobalt Blue The above image shows a picture of mixture pigment, Vermillion with Cobalt Blue, and pure pigments, Cobalt Blue and Vermillion.

Histogram of Pure Cobalt Blue Histogram of Pure Vermillion

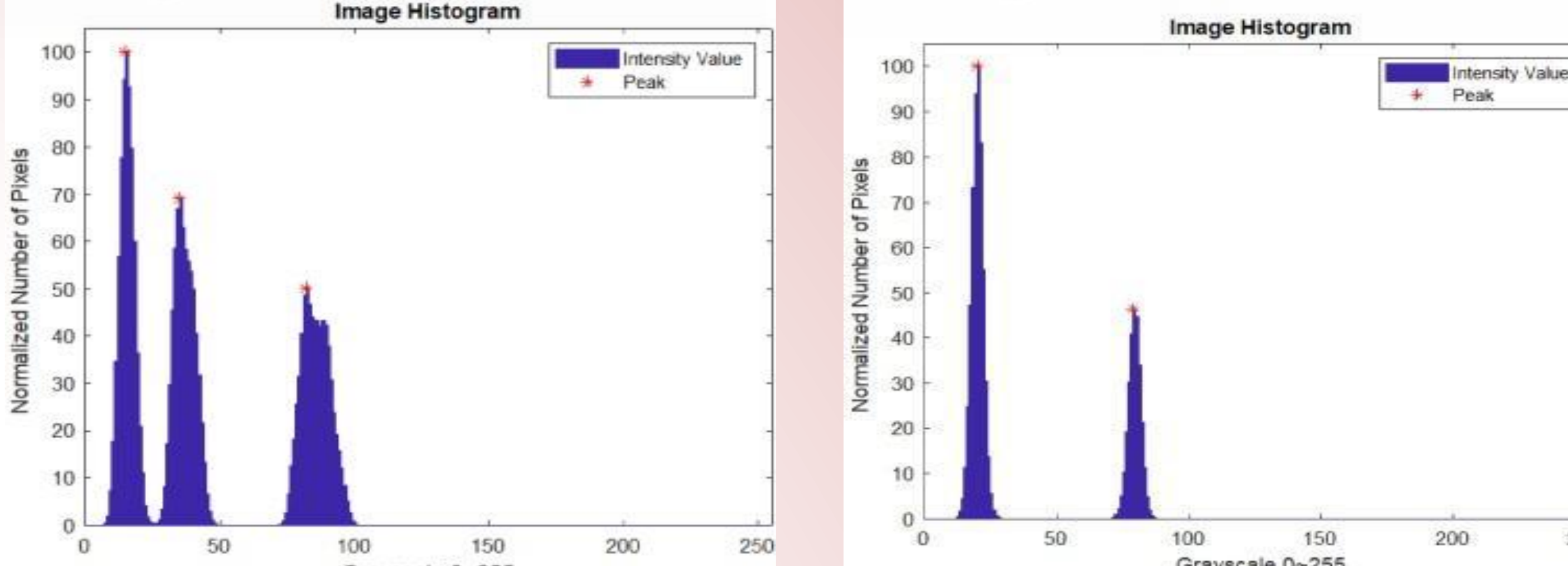

Histogram of a mixture of Cobalt Blue and Vermillion

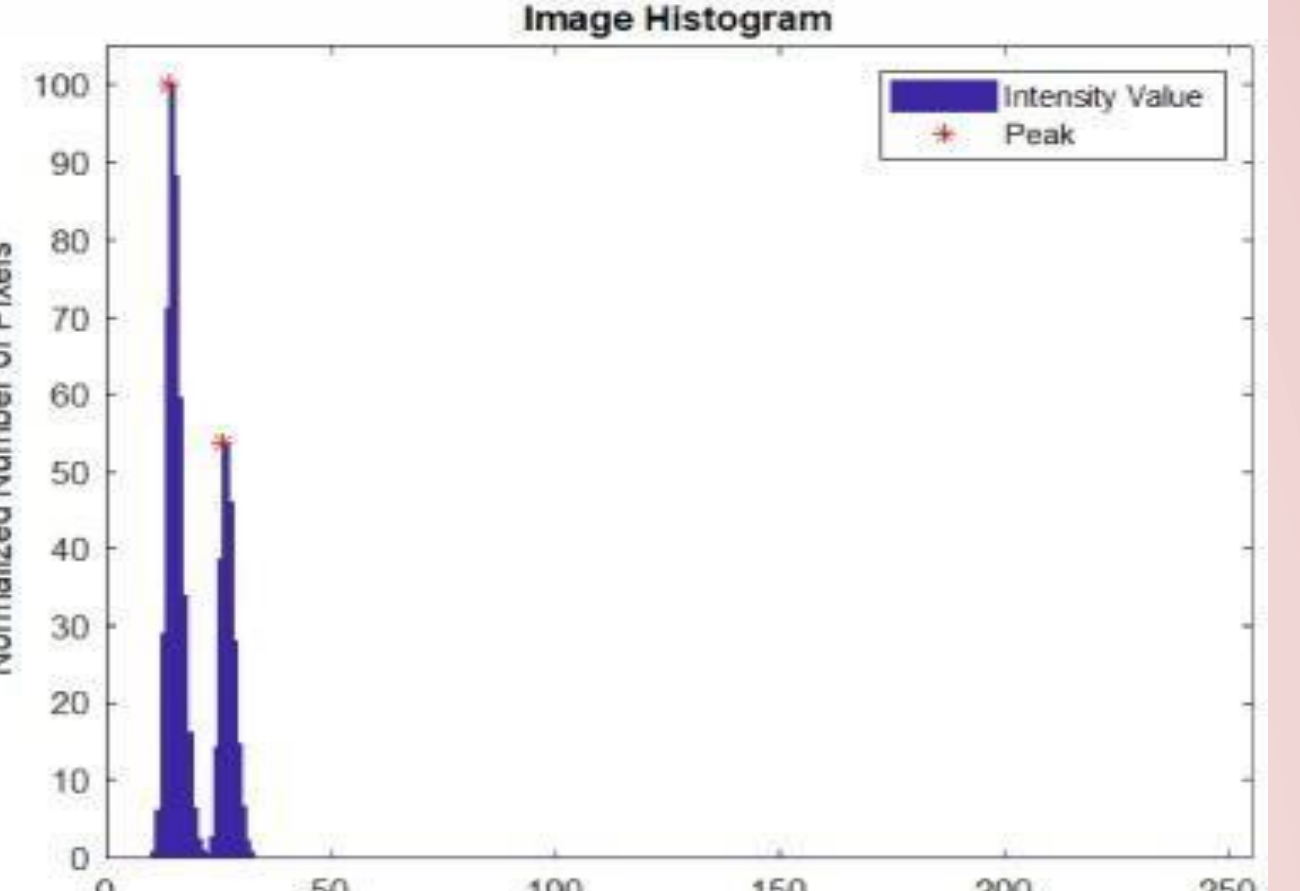

Table of Histogram Peaks Por Pigment Sample

\begin{tabular}{|c|c|}
\hline Name of Pigment & Peak Location in Histogram \\
\hline lizarin Red/Cadmium Yellow & 1542 \\
\hline Alizarin Red & 1230 \\
\hline Alizarin Red/Vermilion & $23 \quad 31 \quad 64$ \\
\hline Cadmium/Naples Yellow & $\begin{array}{lll}4 & 54 & 86\end{array}$ \\
\hline admium Yellow/Lead White & $81 \quad 107$ \\
\hline Cadmium Yellow & 91126 \\
\hline Cobalt Blue & 143481 \\
\hline Cobalt Blue/Titanium White & $82 \quad 163$ \\
\hline Ivory Black & 14 \\
\hline Ivory /Mars Black & $\begin{array}{lll}6 & 13 & 19\end{array}$ \\
\hline Lead White & 138 \\
\hline Lead/Titanium White & $\begin{array}{lll}118 & 151 & 177\end{array}$ \\
\hline Mars Black & 12 \\
\hline Naples Yellow & $21 \quad 61 \quad 87$ \\
\hline ples Yellow/Ultra Marine Blue & 2431 \\
\hline Titanium White & 132146 \\
\hline Titanium White/ Ivory Black & $\begin{array}{lll}30 & 48 & 64\end{array}$ \\
\hline Ultra Marine Blue & $\begin{array}{llll}6 & 13 & 49 & 60\end{array}$ \\
\hline Ultra Marine /Cobalt Blue & $\begin{array}{lll}6 & 19 & 72\end{array}$ \\
\hline Vermillion/Cobalt Blue & 1325 \\
\hline Vermillion & \\
\hline
\end{tabular}

\section{Conclusion}

LIBS and Digital Imaging are useful methods for the detection of pigments either pure or in binary mixtures. Distinguishing between pigments was successful by comparing the characteristic elements present in the individual LIBS spectra. In the case of mixtures, both pigments were identified demonstrating LIBS's ability to detect multiple pigments in overlapping paint layers.

Fr the digital analysis, the pigments' histograms were analyzed and the results showed that pure pigments and the mixture pigments their unique peak locations. Fundamental pigments can be effectively distinguished and separated according to the peak locations of their pigments' histograms The identification of pigments using this method can help to determine authenticity and to date artwork indirectly. Identifying a pigment such as Mars Black or Titanium White would indicate the artwork could not be dated earlier than the 19th century when these whecame cor painting containing a moder pigment is elther a forgery or has been referring to characteristic emission lines in LIBS spectra and to the historical background of the associated pigments.

\section{Relerenees}

P. Hunt, "Revolution in Paint." North Carolina Museum of Art 2006, 1-16. the Applications carried out at FORTH-IESL." Advanced Research Center for Cultural (ARCCHIP) 2009 .

D. Anglos, S. Couris, and C. Fotakis, "Laser Diagnostics of Painted Artworks: Laser-
Induced Breakdown Spectroscopy in Pigment Identification." Applied Spectroscopy

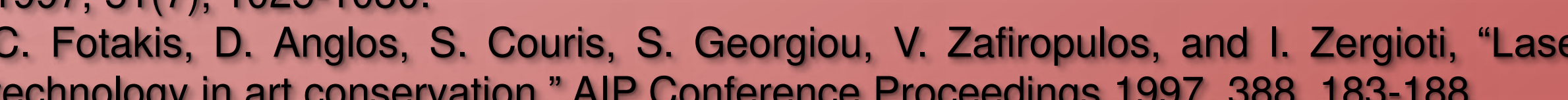
K. Melessanaki, V. Papdakis, C. Balas, and D. Anglos, "Laser induced breakdown
spectroscopy and hyper-spectral imaging analysis of pigments on an illuminated

F. S. L. Borba, J. Cortez, V. K. Asfora, C. Pasquini, M. F. Pim 